\title{
HST NICMOS photometry of the reddened bulge globular clusters NGC 6528, Terzan 5, Liller 1, UKS 1 and Terzan 4*
}

\author{
S. Ortolani ${ }^{1}$, B. Barbuy ${ }^{2}$, E. Bica ${ }^{3}$, A. Renzini ${ }^{4}$, M. Zoccali $^{4}$, R. M. Rich ${ }^{5}$, and S. Cassisi ${ }^{6}$ \\ 1 Università di Padova, Dept. di Astronomia, Vicolo dell'Osservatorio 2, 35122 Padova, Italy \\ e-mail: ortolani@pd.astro.it \\ 2 Universidade de São Paulo, CP 3386, São Paulo 01060-970, Brazil \\ e-mail: barbuy@iagusp.usp.br \\ 3 Universidade Federal do Rio Grande do Sul, Dept. de Astronomia, CP 15051, Porto Alegre 91501-970, Brazil \\ e-mail: bica@if.ufrgs.br \\ 4 European Southern Observatory, Karl Schwarzschild Strasse 2, 85748 Garching bei München, Germany \\ e-mail: arenzini@eso.org \\ 5 Department of Physics and Astronomy, Division of Astronomy and Astrophysics, University of California, \\ Los Angeles, CA 90095-1562, USA \\ e-mail: rmr@astro.ucla.edu \\ ${ }^{6}$ Osservatorio Astronomico Collurania, 64100 Teramo, Italy \\ e-mail: cassisi@astrte.te.astro.it
}

Received 5 June 2001 / Accepted 16 July 2001

\begin{abstract}
We present results from NICMOS Hubble Space Telescope observations of the reddened bulge globular clusters NGC 6528, Terzan 5, Liller 1, UKS 1 and Terzan 4, obtained through the filters F110W (1.1 $\mu \mathrm{m})$ and F160W $(1.6 \mu \mathrm{m})$ (nearly equivalent to $J$ and $H$ ). For the first time the turnoff region of Liller 1 and the main sequence of Terzan 5 and Terzan 4 are reached, as well as the horizontal branch of UKS 1 . The magnitude difference between the turnoff and the red horizontal branch $\Delta m_{110}=m_{110}^{\mathrm{HB}}-m_{110}^{\mathrm{TO}}$ is used as an age indicator. From comparisons with new isochrones in the NICMOS photometric system, we conclude that the two metal-rich clusters NGC 6528 and Terzan 5 are coeval within uncertainties $(\sim 20 \%)$ with 47 Tucanae. Liller 1 and UKS 1 are confirmed as metal-rich globular clusters. Terzan 4 is confirmed as an interesting case of a metal-poor cluster in the bulge with a blue horizontal branch.
\end{abstract}

Key words. Galaxy: globular clusters: individual: NGC 6528, Terzan 5, Liller 1, Terzan 4 UKS 1 - stars: CM diagram

\section{Introduction}

Because they are simple stellar populations of single age and abundance, globular clusters located in the central regions of the Galaxy offer a special opportunity to study the stellar populations in the Galactic bulge (e.g. Minniti 1995; Ortolani et al. 1995; Barbuy et al. 1998). The distribution of cluster metallicities coupled with their spatial location places interesting constraints on the formation

\footnotetext{
Send offprint requests to: M. Zoccali,

e-mail: mzoccali@eso.org

* Based on observations collected with the NASA/ESA Hubble Space Telescope obtained at the Space Telescope Science Institute, which is operated by the Association of Universities for Research in Astronomy, Inc., under NASA contract NAS 5-16555.
}

processes and timescales of the inner spheroid (Ortolani et al. 1995 and references therein). The kinematics of globular clusters within $4 \mathrm{kpc}$ from the Galactic center (as derived from the radial velocities of cluster members) indicates that most of such clusters do indeed belong to the bulge (Côté 1999). Fundamentally, we want to know the ages of these clusters relative to their counterparts in the distant halo, and relative to the field stars in the Galactic bulge.

Many of the bulge globular clusters suffer from significant extinction and some, such as Liller 1, are essentially invisible in the optical. However, even with ground-based infrared detectors, study of these clusters has been difficult: they are extremely crowded, and located in the heavily crowded bulge field. Their great distances have 
Table 1. Reddening and metallicity of the program clusters.

\begin{tabular}{|c|c|c|c|c|c|c|}
\hline \multirow[b]{2}{*}{ Cluster } & \multirow[b]{2}{*}{$l$} & \multicolumn{3}{|c|}{ Barbuy et al. (1998) } & \multicolumn{2}{|c|}{ Harris' compilation } \\
\hline & & $b$ & $E(B-V)$ & {$[\mathrm{M} / \mathrm{H}]$} & $E(B-V)$ & {$[\mathrm{M} / \mathrm{H}]$} \\
\hline Terzan 5 & 3.81 & 1.67 & 2.39 & 0.00 & 2.37 & -0.28 \\
\hline NGC 6528 & 1.14 & -4.18 & 0.52 & -0.2 & 0.56 & -0.17 \\
\hline Terzan 4 & 356.02 & 1.31 & 2.31 & $-2.0:$ & 2.35 & -1.60 \\
\hline Liller 1 & 354.81 & -0.16 & 3.00 & +0.2 & 3.00 & +0.22 \\
\hline UKS 1 & 5.13 & 0.76 & 3.10 & -0.5 & 3.09 & -0.50 \\
\hline
\end{tabular}

prevented efforts to reach the turnoff point from the ground. The extinction is large and spatially variable, so that even in the infrared the main sequences of their colormagnitude diagrams (CMDs) have large scatter. For those clusters in the inner bulge, very high spatial resolution and point spread function stability is required for success, hence our choice to use the NICMOS detector on board HST.

Ortolani et al. (1995) have analysed the relatively low reddening bulge clusters NGC 6528 and NGC 6553 using HST optical $V$ and $I$ photometry, and concluded that their age is close to that of the inner halo cluster 47 Tucanae. NGC 6553 has also been studied in the nearIR ( $J$ and $K$ photometry, Guarnieri et al. 1998), while an HST proper motion study of stars in its field has allowed investigators to largely decontaminate its CMD from foreground/background stars belonging either to the disk or the bulge (Zoccali et al. 2001a); the resulting very clean turnoff point confirms the cluster age.

Terzan 1, a highly reddened globular cluster well within the bulge, has also been studied with HST (Ortolani et al. 1999), revealing that this is a low metallicity cluster with a fairly red horizontal branch, i.e. a second parameter cluster, possibly a few Gyr younger than the majority of globular clusters in the Galaxy.

In the present study we further expand these studies, providing new results based on HST/NICMOS observations of NGC 6528 and of four other among the most reddened bulge and lowest galactic latitute clusters, namely UKS 1, Liller 1, Terzan 4 and Terzan 5. Near-IR observations are the best hope for deriving deep CMDs for these clusters. Even so, we are able to report ages for only 2 of the four clusters.

Reddening and metallicities for the sample clusters (Table 1) have been taken from Barbuy et al. (1998), and the compilation by Harris (1996), as updated in the web page http://physun.physics.mcmaster.ca/ Globular.html. Previous information on these clusters can be summarized as follows.

NGC 6528 is the best studied cluster of our sample, due to its moderate reddening $E(B-V)=0.52$. Its overall metallicity is expected to be $[\mathrm{M} / \mathrm{H}] \approx-0.2 \div 0$. Indeed, the strong similarity between its optical CMD and that of NGC 6553 (Ortolani et al. 1995) suggests that the two clusters have essentially the same metallicity. For NGC 6553 Barbuy et al. (1999) found $[\mathrm{Fe} / \mathrm{H}] \approx-0.55$ from the analysis of two red giant stars, while Cohen et al. (1999) found $[\mathrm{Fe} / \mathrm{H}] \approx-0.17$ from a sample of horizontal branch (HB) stars. Despite the discrepancy in the measured $[\mathrm{Fe} / \mathrm{H}]$ abundances, the two groups found global metallicity values that are compatible within the errors: $[\mathrm{M} / \mathrm{H}] \approx-0.25$ according to Barbuy et al. (1999), and $[\mathrm{M} / \mathrm{H}]=-0.1 \div+0.16$ (depending upon the adopted [O/Fe]) according to Cohen et al. (1999). Near-IR photometry for this cluster has been recently published by Davidge (2000), but it does not reach the main sequence turnoff.

Terzan 4 appeared to have a blue horizontal branch (HB) morphology, detected in a $V, I$ CMD (Ortolani et al. 1997a), obtained under exceptional seeing conditions (0."34). The results suggested a metal-poor bulge cluster.

Terzan 5 is among the most metal-rich globular clusters in the Galaxy, as indicated by its $V, I$ CMD (Ortolani et al. 1996a), and integrated spectra (Bica et al. 1998). The CMD also shows strong differential reddening effects.

Liller 1 is also quite metal rich, Frogel et al. (1995) derived $[\mathrm{Fe} / \mathrm{H}]=+0.25 \pm 0.3$ from $J, H$ and $K$ CMDs. Further evidence of a high metallicity was present in $I$ and Gunn $z$ CMDs of the Red Giant Branch (RGB) (Ortolani et al. 1996b). A nice near-IR CMD of Liller 1, reaching the turnoff magnitude, has been recently obtained by Davidge (2000), but the strong contamination by bulge stars does not allow a reliable measure of the cluster turnoff magnitude.

UKS 1 is the most reddened of the program clusters, only the RGB was so far detected by means of $I, z, J, K$ CMDs (Minniti et al. 1995; Ortolani et al. 1997b). A nearIR integrated spectrum indicates a high metallicity (Bica et al. 1998), and infrared echelle data (Rich et al. 2001) give $[\mathrm{Fe} / \mathrm{H}]=-0.3$ with $[\alpha / \mathrm{Fe}]=+0.3$.

The main purpose of the present paper is to use the minimized reddening effects in the near-IR, combined with the HST/NICMOS image quality to produce highly improved CMDs, helping to determine the cluster ages.

In Sect. 2 the observations and reductions are described. In Sect. 3 the CMDs of the clusters are presented. In Sect. 4 their ages and other properties are discussed. Concluding remarks are given in Sect. 5. 


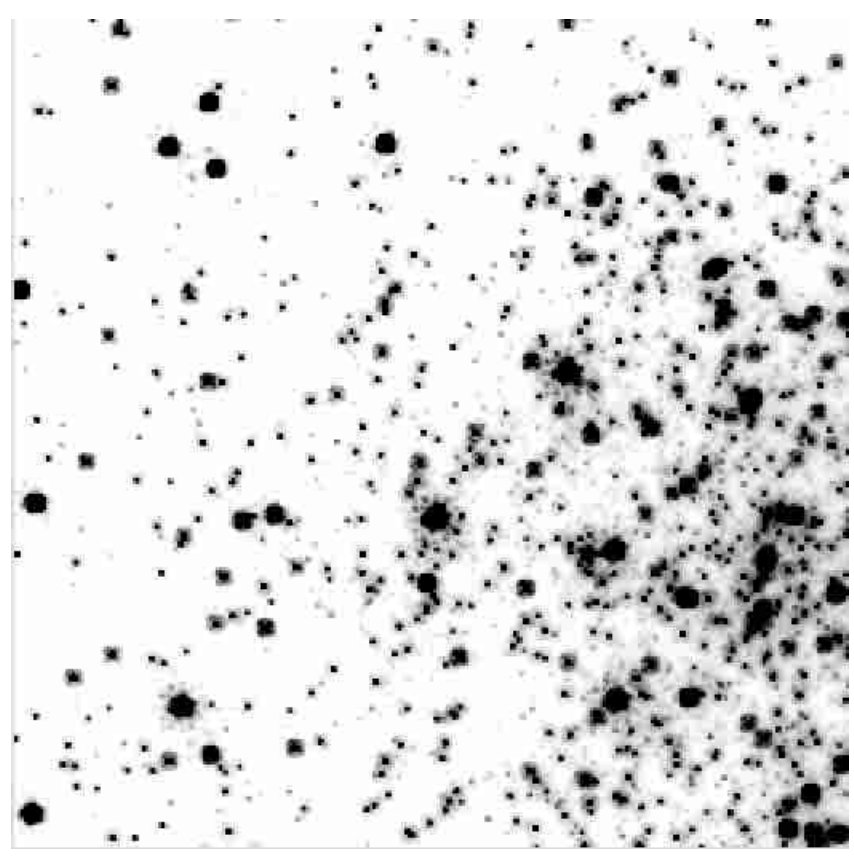

Fig. 1. Terzan 5: F110W NIC2 offset image of the cluster. Dimensions are $19.2^{\prime \prime} \times 19.2^{\prime \prime}$.

\section{Observations and reductions}

The clusters were observed with NICMOS on board HST, through the F110W and F160W filters. Images were taken with NIC1 and NIC2 cameras. The centers of the clusters were in NIC2, used as a primary camera, while parallel observations with NIC1, which points $\sim 30$ arcsec away from NIC2, provided higher resolution on the clusters more external parts. A second pointing of both cameras was taken with NIC2 located at $\sim 20$ arcsec from the center of each cluster, in order to sample less crowded regions. All exposures were obtained using the MULTIACCUM readout mode and the STEP64 time sequence through four dithering positions. Exposure times are reported in the log of observations (Table 2).

The pixel size of NIC2 is $0 . \prime 075$, giving a field of view of $19^{\prime \prime} 2 \times 19^{\prime \prime} 2$ for each frame, while NIC1 samples 0 "' 043 per pixel, for a total field of $11^{\prime \prime} \times 11^{\prime \prime}$. In Fig. 1 we show, as an example, a F110W NIC2 image of Terzan 5. Note the high angular resolution obtained.

The images were preprocessed by the standard NICMOS pipelines CALNICA and CALNICB. These routines produce a single background-subtracted mosaic image expressed in counts/sec/pixel. DAOPHOT II and ALLSTAR (Stetson 1987) were used to determine the instrumental magnitudes. The typical $F W H M$ of the stars was $0 . \prime 11$. We determined the aperture corrections from isolated stars and applied them to derive the stellar magnitudes within a 0.55 aperture and normalized to nominal infinite aperture. The magnitudes were then converted to count rates and multiplied by the inverse sensitivity given as the keyword PHOTFLAM in the header of the images. Finally, the zero point PHOTZPT was applied
Table 2. Log of observations.

\begin{tabular}{|c|c|c|c|c|c|}
\hline Cluster & Camera & Field & Filter & Date & Exp.(s) \\
\hline Terzan 4 & NIC2 & CEN & F110W & 27-04-98 & 256 \\
\hline$"$ & $"$ & CEN & F160W & 27-04-98 & 320 \\
\hline$"$ & $"$ & $\mathrm{OFF}$ & F160W & $27-04-98$ & 352 \\
\hline$"$ & $"$ & $\mathrm{OFF}$ & F110W & 27-04-98 & 256 \\
\hline$"$ & NIC1 & CEN & F110W & 27-04-98 & 256 \\
\hline$"$ & $"$ & CEN & F160W & 27-04-98 & 320 \\
\hline$"$ & $"$ & $\mathrm{OFF}$ & F110W & $27-04-98$ & 256 \\
\hline$"$ & $"$ & $\mathrm{OFF}$ & F160W & $27-04-98$ & 352 \\
\hline Terzan 5 & NIC2 & CEN & F110W & $23-04-98$ & 256 \\
\hline$"$ & $"$ & CEN & F160W & $23-04-98$ & 320 \\
\hline$"$ & $"$ & $\mathrm{OFF}$ & F110W & $23-04-98$ & 256 \\
\hline$"$ & $"$ & $\mathrm{OFF}$ & F160W & $23-04-98$ & 352 \\
\hline$"$ & NIC1 & CEN & F110W & 23-04-98 & 256 \\
\hline$"$ & $"$ & CEN & F160W & $23-04-98$ & 320 \\
\hline$"$ & $"$ & $\mathrm{OFF}$ & F110W & $23-04-98$ & 256 \\
\hline$"$ & $"$ & $\mathrm{OFF}$ & F160W & $23-04-98$ & 352 \\
\hline NGC 6528 & $\mathrm{NIC} 2$ & CEN & F110W & $17-04-98$ & 256 \\
\hline$"$ & $"$ & CEN & F160W & $17-04-98$ & 320 \\
\hline$"$ & $"$ & $\mathrm{OFF}$ & F110W & $17-04-98$ & 256 \\
\hline$"$ & $"$ & $\mathrm{OFF}$ & F160W & $17-04-98$ & 352 \\
\hline$"$ & NIC1 & CEN & F110W & $17-04-98$ & 256 \\
\hline$"$ & $"$ & CEN & F160W & $17-04-98$ & 320 \\
\hline$"$ & $"$ & $\mathrm{OFF}$ & F110W & $17-04-98$ & 256 \\
\hline$"$ & $"$ & OFF & F160W & $17-04-98$ & 352 \\
\hline UKS 1 & $\mathrm{NIC} 2$ & CEN & F110W & 06-03-98 & 256 \\
\hline 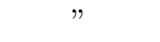 & $"$ & CEN & F160W & 06-03-98 & 320 \\
\hline$"$ & $"$ & $\mathrm{OFF}$ & F110W & 06-03-98 & 256 \\
\hline$"$ & $"$ & $\mathrm{OFF}$ & F160W & 06-03-98 & 352 \\
\hline$"$ & NIC1 & CEN & F110W & 06-03-98 & 256 \\
\hline$"$ & $"$ & $\mathrm{CEN}$ & F160W & $06-03-98$ & 320 \\
\hline$"$ & $"$ & $\mathrm{OFF}$ & F110W & 06-03-98 & 256 \\
\hline$"$ & $"$ & $\mathrm{OFF}$ & F160W & 06-03-98 & 352 \\
\hline Liller 1 & NIC2 & CEN & F110W & 08-08-98 & 256 \\
\hline 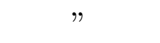 & $"$ & CEN & F160W & 08-08-98 & 320 \\
\hline$"$ & $"$ & $\mathrm{OFF}$ & F110W & 08-08-98 & 256 \\
\hline$"$ & $"$ & $\mathrm{OFF}$ & F160W & 08-08-98 & 352 \\
\hline$"$ & NIC1 & $\mathrm{CEN}$ & F110W & $08-08-98$ & 256 \\
\hline$"$ & $"$ & CEN & F160W & 08-08-98 & 320 \\
\hline$"$ & $"$ & OFF & F110W & 08-08-98 & 256 \\
\hline$"$ & $"$ & $\mathrm{OFF}$ & F160W & 08-08-98 & 352 \\
\hline
\end{tabular}

to transform instrumental magnitudes into the standard HST $m_{110}$ and $m_{160}$ magnitudes used hereafter.

The presently available empirical transformations between NICMOS/HST $m_{110}$ and $m_{160}$ and standard ground-based $J$ and $H$ (Stephens et al. 2000) are not suitable for the wide range of colours covered by our observations. Instead, we prefer to use the theoretical transformations derived by Origlia \& Leitherer (2000) to convert theoretical isochrones into the HST/NICMOS observational plane.

\section{Colour magnitude diagrams}

Figures 2 to 6 show the $m_{110}$ vs. $m_{110}-m_{160}$ CMDs of NGC 6528, Terzan 5, Liller 1, UKS 1 and Terzan 4. Each figure contains $4 \mathrm{CMDs}$ of a given cluster, corresponding 


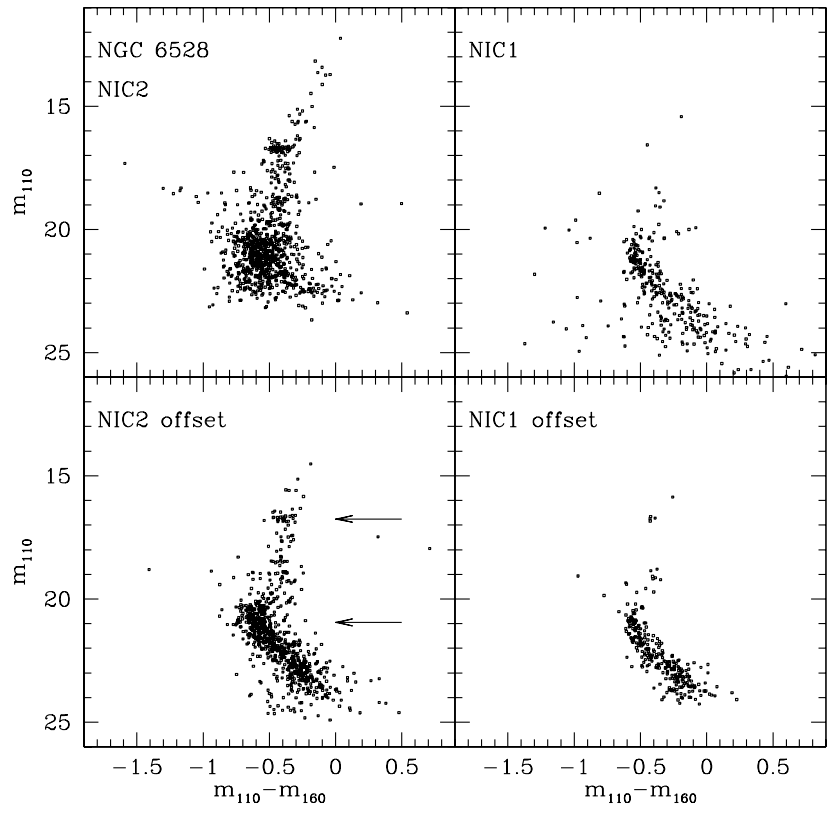

Fig. 2. CMD of the four fields observed in NGC 6528. The upper left panel refers to the cluster center region. The arrows show the estimated positions of HB and turnoff.

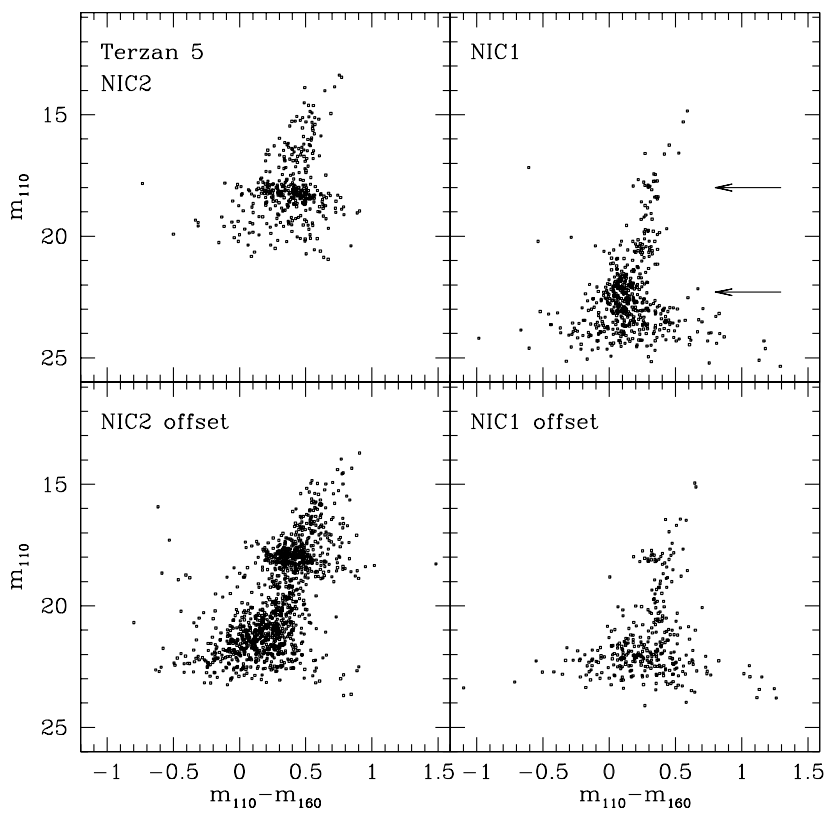

Fig. 3. CMD of the four fields observed in Terzan 5. The upper left panel refers to the cluster center region. The arrows show the estimated positions of HB and turnoff.

to the four pointings described above and indicated in the figure labels. NIC2 has a wider field so that the corresponding panels show more populated cluster sequences, in particular the HB. On the other hand, the NIC1 camera allowed us to obtain more accurate photometry and deeper diagrams due to its smaller pixel size, giving a better PSF sampling, hence reaching fainter magnitudes, especially in the most crowded regions.

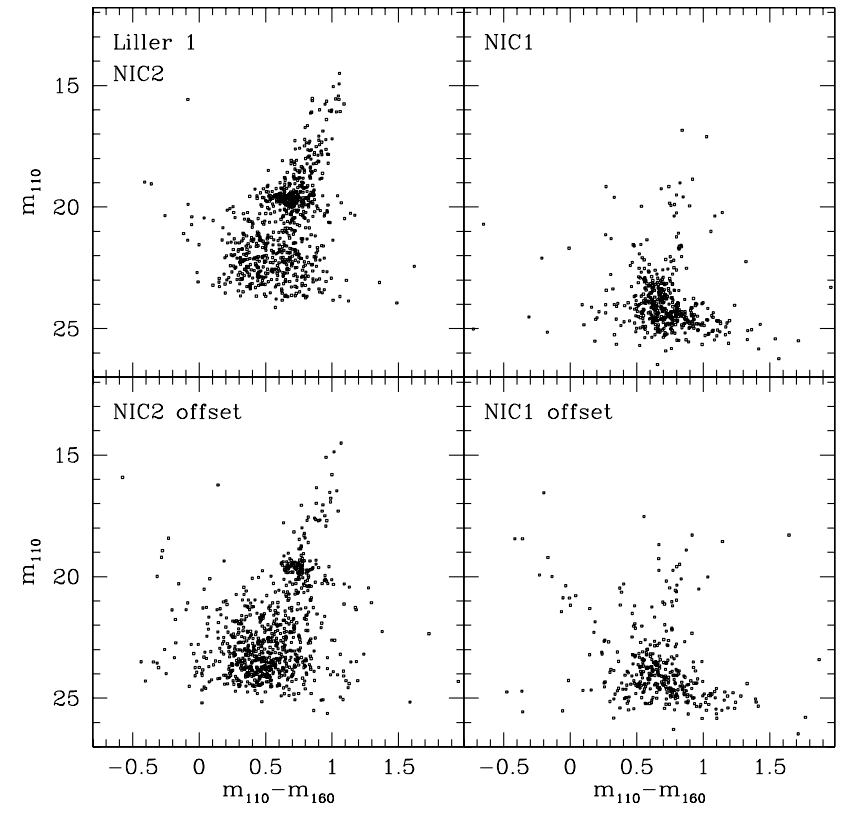

Fig. 4. CMD of the four fields observed in Liller 1. The upper left panel refers to the cluster center region.

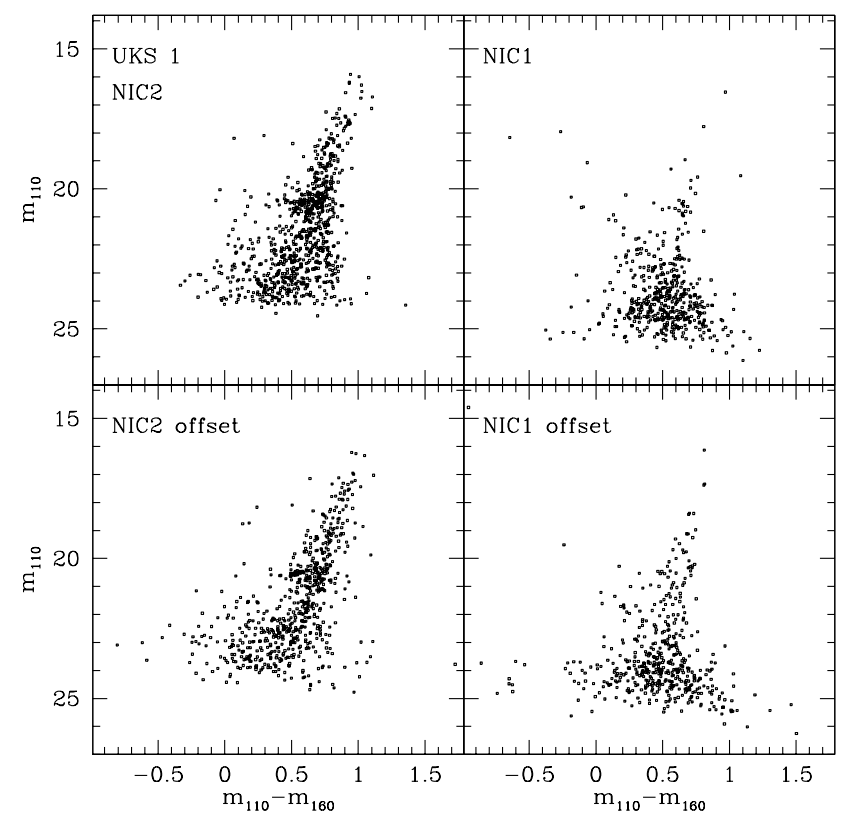

Fig. 5. CMD of the four fields observed in UKS 1. The upper left panel refers to the cluster center region.

The CMDs of NGC 6528 (Fig. 2) reach more than 3 mag below the turnoff in the two NIC1 and the NIC2offset pointings.

The deepest CMD for Terzan 5, reaching about $1 \mathrm{mag}$ below the turnoff, is given in the upper right panel of Fig. 3. The brighter cluster sequences are well populated in the NIC2 CMDs. The reduced effects of differential reddening are noticeable with respect to the optical CMDs (Ortolani et al. 1996a).

The red HB morphology of Liller 1 (Fig. 4) is clearly seen for the first time. Moreover the NIC1 CMDs have shown the cluster turnoff, which is an important result 


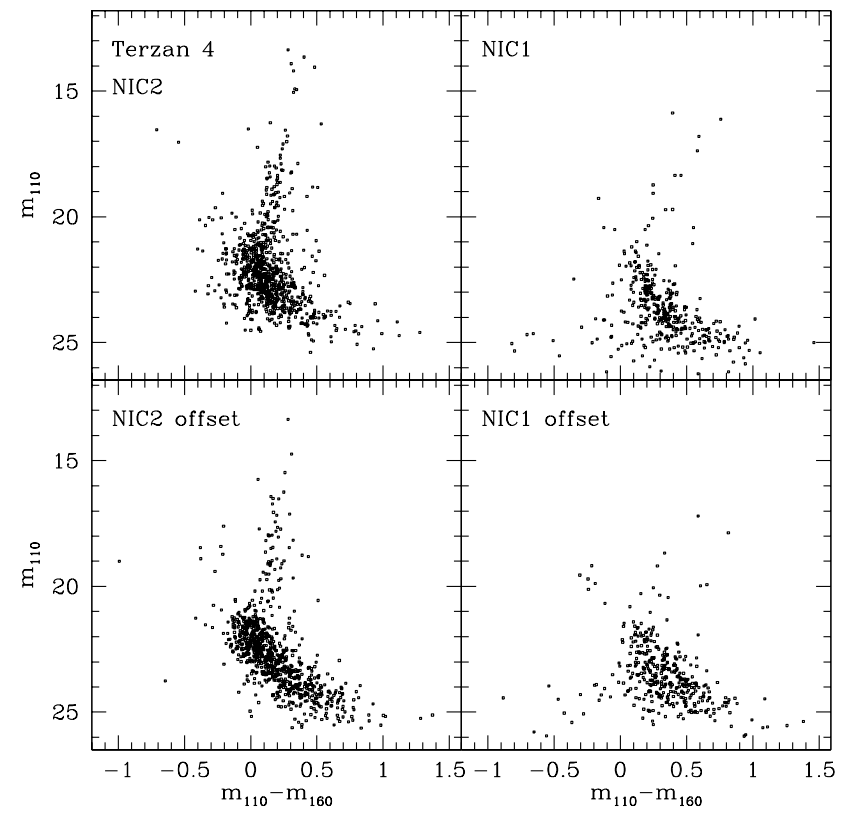

Fig. 6. CMD of the four fields observed in Terzan 4. The upper left panel refers to the cluster center region.

for such a low Galactic latitude and very reddened bulge cluster. The red HB confirms the high metallicity, as also derived by Frogel et al. (1995) from the RGB slope in the infrared CMD.

The HB morphology of UKS 1 (Fig. 5) is seen for the first time. The red HB confirms that the cluster is metal rich (Bica et al. 1998). Since the reddening values of UKS 1 and Liller 1 are quite similar (see references in Sect. 1), the fainter HB of UKS 1 implies that it is more distant. As a result the UKS 1 CMDs barely reach the SGB region.

Terzan 4 (Fig. 6) CMDs are very deep attaining more than 3 mag below the turnoff in both cameras. Note the absence of a red HB, suggesting presence of a sparsely populated blue $\mathrm{HB}$, which, in these bands, would be located at $m_{110}-m_{160} \sim-0.3$ (see the two lower panels of Fig. 6). The metal poor nature of this cluster (Ortolani et al. 1997a) is therefore confirmed.

\section{Cluster ages}

The magnitude difference between the HB and the turnoff $\Delta V_{\mathrm{TO}}^{\mathrm{HB}}$ is an effective age indicator (e.g., Iben 1974; Sandage 1986), and the method has been widely used, especially to derive relative ages (e.g. Ortolani et al. 1995, 1999; Chaboyer et al. 1996; Rosenberg et al. 1999). In particular, the method was applied to constrain in a fully empirical way any age difference between the bulge globulars NGC 6528 and NGC 6553 and the halo globular clusters (Ortolani et al. 1995).

While most of these studies used optical photometry, we now adopt the same approach using the NICMOS nearIR data. From the CMDs presented in the previous section accurate values of $\Delta m_{110}=m_{110}^{\mathrm{HB}}-m_{110}^{\mathrm{TO}}$ can be obtained only for NGC 6528 and Terzan 5. In Figs. 2 and 3 the HB and turnoff locations are indicated by arrows. The resulting $\Delta m_{110}$ are reported in Table 3 together with the estimated uncertainty. Unfortunately, the CMDs for Liller 1 and UKS 1 are not accurate enough for a similar measure: the turnoff is not very well defined, and it might appear brighter than it is due to crowding (blends). Moreover, for these clusters the turnoff and the HB were not measurable from a single camera, and possible residual offsets between NIC1 and NIC2 may introduce a systematic error. In the case of Terzan 4 the turnoff is fairly well defined, but the blue morphology together with the sparse population of the HB makes it impossible to derive the corresponding value of $\Delta m_{110}$. The value of $\Delta m_{110}$ for 47 Tuc was also measured from a CMD obtained from NIC3 archive data (program GO7318) taken through the same filters. The result is also reported in Table 3 and will be used later to constrain the age difference, if any, between the bulge clusters and this inner halo cluster. The overall metallicity of 47 Tuc was adopted to be $[\mathrm{M} / \mathrm{H}]=-0.5$, given its iron abundance $[\mathrm{Fe} / \mathrm{H}]=-0.7$ and $\alpha$-element enhancement $[\alpha / \mathrm{Fe}] \simeq 0.3$ (Gratton et al. 1986).

\subsection{Isochrones in the NICMOS $m_{110}-m_{160}$ system}

To further constrain the ages of the program clusters, new isochrones in the NICMOS photometric system were constructed. All the evolutionary tracks used for producing the set of isochrones adopted in the present analysis have been obtained by means of the FRANEC evolutionary code (Cassisi \& Salaris 1997; Castellani et al. 1999). The input physics includes the OPAL radiative opacities (Iglesias \& Rogers 1996) for temperatures higher than $10000 \mathrm{~K}$, while for lower temperatures the molecular opacity tables provided by Alexander \& Ferguson (1994) have been adopted. This choice allows us to have a smooth match between the two different opacity sets. Both high and low-temperature opacities have been computed by assuming a solar-scaled heavy elements distribution (Grevesse 1991). The Straniero (1988) equation of state was adopted, while superadiabatic convection was treated in the mixing length approximation with calibration provided by Salaris \& Cassisi (1996). More on the input physics can be found in Cassisi \& Salaris (1997). The evolutionary computations do not consider atomic diffusion. However, it is well known (Castellani et al. 1997; Cassisi et al. 1998) that accounting for such a mechanism leads to a reduction of the cluster ages by $\sim 0.7-1$ Gyr.

Stellar models and isochrones have been constructed for the following initial chemical abundances: $(Z=$ $0.006, Y=0.25)$ and $(Z=0.02, Y=0.289)$, which assume a helium enrichment ratio $\mathrm{d} Y / \mathrm{d} Z=2.8$. For each adopted chemical composition, Zero Age Horizontal Branch (ZAHB) models corresponding to a RGB progenitor with initial mass equal to $1 M_{\odot}$ have been computed. In fact, the ZAHB structures have been constructed for each fixed metallicity by using the helium core mass and the envelope chemical abundance profile suitable for a 
Table 3. Comparison of $\Delta m_{110}$ measured on the CMDs and in theoretical isochrones. A ratio $\mathrm{d} Y / \mathrm{d} Z=2.8$ has been assumed in these models, see discussion in the text.

\begin{tabular}{lrcccc}
\hline Object & {$[\mathrm{M} / \mathrm{H}]$} & Age $(\mathrm{Gyr})$ & $m_{110}^{\mathrm{HB}}$ & $m_{110}^{\mathrm{TO}}$ & $\Delta m_{110}$ \\
\hline 47 Tuc & -0.50 & $15 \pm 3$ & 16.50 & 20.70 & $4.2 \pm 0.20$ \\
Terzan 5 & 0.00 & $14 \pm 3$ & 18.00 & 22.30 & $4.3 \pm 0.20$ \\
NGC 6528 & -0.20 & $13 \pm 3$ & 16.75 & 20.95 & $4.2 \pm 0.15$ \\
Terzan 4 & -2.00 & & & 21.90 & \\
Liller 1 & +0.20 & & 19.60 & & \\
UKS 1 & -0.50 & & 20.50 & & \\
model & 0.00 & 10 & & & 4.13 \\
model & 0.00 & 12 & & & 4.22 \\
model & 0.00 & 14 & & & 4.30 \\
model & 0.00 & 16 & & & 4.37 \\
model & -0.50 & 10 & & & 3.86 \\
model & -0.50 & 12 & & & 4.06 \\
model & -0.50 & 14 & & & 4.14 \\
model & -0.50 & 15 & & & 4.20 \\
model & -0.50 & 16 & & & 4.25 \\
model & -0.50 & 17 & & & 4.30 \\
\hline
\end{tabular}

RGB progenitor with this mass. All the isochrones have been transferred from the theoretical plane to the observational one in the HST/NICMOS photometric system (F110W, F160W and F222W filters) using recent prescriptions (Origlia \& Leitherer 2000). The resulting $\Delta m_{110}$ values for ages in the range $7 \leq t \leq 17$ (Gyr) are listed in Table 3.

\subsection{Results}

We first proceed differentially, comparing the $\Delta m_{110}$ values of the program clusters to that of the reference cluster 47 Tuc. As reported in Table 3 , the values of $\Delta m_{110}$ for Terzan 5 and NGC 6528 are identical to the value for 47 Tuc, well within the $\pm 0.2 \mathrm{mag}$ uncertainty. There are, however, metallicity differences by $\sim 0.2$ and $\sim 0.3$ dex among the three clusters that must be taken into account. From the theoretical values also listed in Table 3 we see that a 0.25 dex difference in metallicity results in a variation by $\sim 0.08 \mathrm{mag}$ in $\Delta m_{110}$, at fixed age.

As a rule of thumb, to a good approximation, cluster relative age errors (variations) are identical to errors (variations) in the turnoff magnitude, i.e., $\delta t / t \approx \delta M_{\mathrm{TO}}$ (e.g., Renzini 1991). Since the HB luminosity is virtually age independent, this implies that cluster age errors (variations) are identical to errors (variations) in $\Delta m_{110}$. Having determined the distance to 47 Tuc using the white dwarf method, Zoccali et al. (2001b) derive an age of $13 \pm 2.5 \mathrm{Gyr}$ and $14 \pm 2.5 \mathrm{Gyr}$ for this cluster, using respectively models with and without diffusion. Given the values of $\Delta m_{110}$ reported in Table 3, and that any effect of metallicity differences among the clusters is obliterated by the error on the $\Delta m_{110}$ values, we conclude that the three clusters are coeval, within a $\pm 20 \%$ uncertainty, or 13 (14) \pm 2.6 Gyr, the value in parenthesis referring to models neglecting diffusion.

As a second approach, we proceed using the full theoretical age-metallicity- $\Delta m_{110}$ relation as reported in Table 3. By interpolating in both $\Delta m_{110}$ and $[\mathrm{M} / \mathrm{H}]$ one then derives an age of $15,15.5$, and $13 \mathrm{Gyr}$, respectively for 47 Tuc, Terzan 5, and NGC 6528. Being all affected by a $\sim 20 \%$ uncertainty, we do not consider as significant these age differences, and once more conclude that no evidence exists for an age difference between these halo and bulge clusters.

These results depend somewhat on the adopted helium-enrichment ratio $(\mathrm{d} Y / \mathrm{d} Z \approx 2.8)$. However, the exact value of this parameter is still a matter of debate. Recent works by Sandquist (2000) and Zoccali et al. (2000) on helium indicators in galactic globular clusters suggest that $\mathrm{d} Y / \mathrm{d} Z<2.5$. Given that, for each fixed cluster age, the luminosity of both the turnoff and the ZAHB strongly depend on the He abundance, we have estimated how much the present results on the cluster ages are affected by our choice on $\mathrm{d} Y / \mathrm{d} Z$. By performing several numerical experiments, we have verified that, at solar metallicity and ages of the order of $10 \mathrm{Gyr}$, the difference in $m_{110}$ magnitude between the turnoff and the reddest point along the theoretical ZAHB depends on the initial He content as: $\Delta m_{110} / \mathrm{d} Y \approx 2.9$. This means that the $\Delta m_{110}$ values shown in Table 3 , for solar metallicity, have to be decreased by about 0.11 mag at each fixed age, if an initial He content $Y=0.25$ is assumed, i.e., the same adopted for the isochrones for the models at metallicity $[\mathrm{M} / \mathrm{H}]=-0.5$. This would increase the age of NGC 6528 and Terzan 5 to about $14 \pm 3$ and $16 \pm 3 \mathrm{Gyr}$, respectively.

It is worth recalling that the absolute ages determined here may be overestimated by $\sim 1$ Gyr, due to the fact that the HB level has been measured in the observed CMD as the mean locus of the HB stars, which is about $\approx 0.1 \mathrm{mag}$ brighter than the ZAHB level given by the theoretical models. We did not try to simulate HB evolution in our models, nor to estimate the lower envelope of the empirical HB distribution because in any case the magnitude spread of the observed $\mathrm{HB}$, mainly due to differential reddening, does not allow precision in the ages better than $\sim 3$ Gyr.

Despite this, the data constrain the age of these clusters to be old (like 47 Tuc), compatible with halo globular cluster ages and confirming the previous HST optical results on the metal-rich bulge clusters NGC 6528 and NGC 6553 (Ortolani et al. 1995).

\section{Concluding remarks}

The magnitude difference between the horizontal branch and the turnoff $\Delta m_{110}$, combined with newly transformed stellar evolution models, gives relative and absolute ages for the clusters 47 Tuc, NGC 6528 and Terzan 5. Within typical $\sim 20 \%$ uncertainty, the bulge clusters NGC 6528 and Terzan 5 appear to be coeval with 47 Tuc and halo globular clusters. In the case of NGC 6528 the results by Ortolani et al. (1995) based on HST $V$ and $I$ 
photometry are confirmed, suggesting that the bulge is as old as NGC 6528 (and 47 Tuc).

The NICMOS CMDs presented here for the clusters Liller 1, UKS 1 and Terzan 4 are the deepest so far obtained for these clusters that in the optical are affected by 7-9 magnitudes of extinction. These unprecedented observations reveal the red horizontal branch morphology of Liller 1 and UKS 1 , and reach the turnoff of these clusters, albeit with insufficient accuracy to allow a meaningful determination of $\Delta m_{110}$. Both the red horizontal branch morphology and the slope of the RGB suggest that UKS 1 is a metal-rich cluster. In the less reddened cluster Terzan 4 the turnoff region is well delineated, but its sparse population and blue horizontal branch prevent us from estimating $\Delta m_{110}$. However we confirm that Terzan 4 has the blue horizontal branch characteristic of a very metal poor cluster.

Acknowledgements. We acknowledge partial financial support from the Brazilian agencies $\mathrm{CNPq}$ and Fapesp. SO acknowledges Italian Ministero dell'Università e della Ricerca Scientifica e Tecnologica (MURST) under the program on "Stellar Dynamics and Stellar Evolution in Globular Clusters: a Challenge for New Astronomical Instruments". SC thanks for the financial support by MURST - Cofin2000 - under the scientific project "Stellar Observables of Cosmological Relevance" (Italy). Support for R.M.R. was provided by NASA through grant number GO-7832 from the Space Telescope Science Institute, which is operated by AURA, Inc., under NASA contract NAS5-26555.

\section{References}

Alexander, D. R., \& Ferguson, J. W. 1994, ApJ, 437, 879 Barbuy, B., Bica, E., \& Ortolani, S. 1998, A\&A, 333, 117

Barbuy, B., Renzini, A., Ortolani, S., Bica, E., \& Guarnieri, M. D. 1999, A\&A, 341, 539

Bica, E., Clariá, J. J., Piatti, A. E., \& Bonatto, C. 1998, A\&AS, 131,483

Cassisi, S., \& Salaris, M. 1997, MNRAS, 285, 593

Cassisi, S., Castellani, V., Degl'Innocenti, S., \& Weiss, A. 1998, A\&AS, 129, 267

Castellani, V., Ciacio, F., Degl'Innocenti, S., \& Fiorentini, G. 1997, A\&AS, 322, 801

Castellani, V., Degl'Innocenti, S., Fiorentini, G., Lissia, M., \& Ricci, B. 1999, Phys. Rep., 281, 309
Chaboyer, B., Demarque, P., Kernan, P. J., \& Krauss, L. M. 1996, Science, 271, 957

Coelho, P., Barbuy, B., Perrin, M.-N., et al. 2001, A\&A, in press [astro-ph/0107219]

Cohen, J. G., Gratton, R. G., Behr, B., \& Carretta, E. 1999, ApJ, 523, 739

Côté, P. 1999, AJ, 118, 406

Davidge, T. J. 2000, ApJS, 126, 105

Frogel, J. A., Kuchinski, L. E., \& Tiede, G. P. 1995, AJ, 109, 1154

Grevesse, N. 1991, IAU Symp. 145, Evolution of Stars: the Photospheric Abundance Connection, ed. G. Michaud, \& A. Tutukov (Dordrecht: Kluwer), 63

Gratton, R. G., Quarta, M. L., \& Ortolani, S. 1986, A\&A, 169, 208

Guarnieri, M. D., Ortolani, S., Montegriffo, P., et al. 1998, A\&A, 331, 70

Harris, W. E. 1996, AJ, 112, 1487

Iben, I. Jr. 1974, ARA\&A, 12, 215

Iglesias, C. A., \& Rogers, F. J. 1996, ApJ, 464, 943

Minniti, D. 1995, AJ, 109, 1663

Minniti, D., Olszewski, E., \& Rieke, M. 1995, AJ, 110, 1686

Origlia, L., \& Leitherer, C. 2000, AJ, 119, 2018

Ortolani, S., Renzini, A., Gilmozzi, et al. 1995, Nature, 377, 701

Ortolani, S., Barbuy, B., \& Bica, E. 1996a, A\&A, 308, 733

Ortolani, S., Bica, E., \& Barbuy, B. 1996b, A\&A, 306, 134

Ortolani, S., Barbuy, B., \& Bica, E. 1997a, A\&A, 319, 850

Ortolani, S., Bica, E., \& Barbuy, B. 1997b, A\&AS, 126, 319

Ortolani, S., Barbuy, B., Bica, E., et al. 1999, A\&A, 350, 840

Renzini, A. 1991, in Observational Tests of Cosmological Inflation, ed. T. Shanks, A. J. Banday, \& R. S. Ellis (Dordrecht: Kluwer), 131

Rich, R. M., Origlia, L., \& Castro, S. M. 2001, in preparation Rosenberg, A., Saviane, I., Piotto, G., \& Aparicio, A. 1999, AJ, 118, 2306

Salaris, M., \& Cassisi, S. 1996, A\&A, 305, 858

Sandage, A. 1986, ARA\&A, 31, 345

Sandquist, E. L. 2000, MNRAS, 313, 571

Stephens, A. W., Frogel, J. A., Ortolani, S., et al. 2000, AJ, 119,419

Stetson, P. B. 1987, PASP, 99, 191

Straniero, O. 1988, A\&AS, 76, 157

Zoccali, M., Cassisi, S., Bono, G., et al. 2000, ApJ, 538, 289

Zoccali, M., Renzini, A., Ortolani, S., Bica, E., \& Barbuy, B. 2001a, AJ, 121, 2638

Zoccali, M., Renzini, A., Ortolani, S., et al. 2001b, ApJ, 553, 733 